\section{Preventing Vascular Catheter-Related Infections: Current Controversies}

Dr. Barry Farr from the University of Virginia Health Sciences Center, Charlottesville, has reviewed studies on the prevention of vascular catheter-related infection. This review focuses on salient controversies regarding optimal preventive methods. Intensity of surveillance for nosocomial infections was the single most important predictor of prevention in the Study of the Efficacy of Nosocomial Infection Control (SENIC). Used suboptimally by most hospitals in the SENIC, surveillance is probably conducted even less today. There has been one randomized trial of the optimal method of aseptic insertion for central venous catheters and none comparing the two most frequently used sites. Scheduled replacement did not prevent infection in multiple randomized trials, but, according to a recent survey, was still being used frequently. Chlorhexidine preparation of skin before and during catheterization has significantly reduced colonization of catheters in multiple randomized trials and should be used.

Impregnation of catheter surfaces, hub surfaces, or both with antiseptics raises less concern about fostering the development of antibiotic resistance than does the use of antibiotics for this purpose.

FROM: Farr BM. Preventing vascular catheter-related infections: current controversies. Clin Infect Dis 2001;33:1733-1738.

\section{MRSA Outbreak in a Burn Unit and the Role of Hydrotherapy Equipment}

Embil et al. from the Health Sciences Centre in Winnipeg, Canada, have reported a multi-institution outbreak caused by a single strain of methicillin-resistant Staphylococcus aureus (MRSA). Between September 19 and November 20, 1996, an index case and 5 secondary cases of nosocomial MRSA occurred on a 26-bed adult plastic surgery and burn unit (PSBU) at a tertiary care teaching hospital. Between November 11 and December 23, 1996, 6 additional cases were identified at a community hospital. One of the community hospital cases was transferred from the PSBU. All strains were identical by pulsed-field gel electrophoresis. MRSA may have contributed to skin graft breakdown in 1 case and delayed wound healing in others. Patients required 2 to 226 isolation days.

A hand-held shower and stretcher for showering in the hydrotherapy room of the PSBU had positive results on culture for the outbreak strain, and the presumed means of transmission. Replacement of stretcher showering with bedside sterile burn wound compresses terminated the outbreak. The PSBU was closed to new admissions and transfers out for 11 days during the investigation. Seven of 12 patients had effective decolonization therapy.

The authors concluded that environmental contamination is a potential source of nosocomial MRSA transmission on a burn unit. Notification among institutions and community care providers of shared patients infected or colonized with an antimicrobial-resistant microorganism is necessary.

FROM: Embil JM, McLeod JA, Al-Barrak AM, et al. An outbreak of methicillin-resistant Staphylococcus aureus on a burn unit: potential role of contaminated hydrotherapy equipment. Burns 2001;27:681-688.

\section{Prolonged Colonization and Clearance of VRE in Long-Term Care}

Baden et al. from Beth Israel Deaconess Medical Center, Boston, Massachusetts, conducted a study on the persistence of colonization with vancomycin-resistant Enterococcus faecium (VRE) in the non-oncologic, non-intensive care unit patient. They studied all patients who had VRE isolated on 2 or more occasions of more than 1 year apart (study A) and those who had been "cleared" of VRE colonization after negative results on 3 stool cultures (study B). Twelve patients had stored VRE isolates recovered more than 1 year apart (study A), and $58 \%$ of paired isolates were genotypically related according to pulsed-field gel electrophoresis patterns. In study B, stool samples were obtained weekly from 21 "cleared" patients for 5 weeks, which revealed that $24 \%$ were VRE positive. For these culture-positive patients, $72 \%$ of the cultures failed to detect VRE.

Recent antibiotic use was significantly more common in the culture-positive patients than in the culture-negative patients $(P=.003)$. Colonization with VRE may persist for years, even if the results of intercurrent surveillance stool and index site cultures are negative. Cultures for detection of VRE in stool samples obtained from patients declared "cleared" are insensitive.

FROM: Baden LR, Thiemke W, Skolnik A, et al. Prolonged colonization with vancomycin-resistant Enterococcus faecium in long-term care patients and the significance of "clearance." Clin Infect Dis 2001;33:1654-1660.

\section{Infected Arteriovenous Grafts in Hemodialysis Patients}

Minga et al. from the University of Alabama at Birmingham identified prospectively all arteriovenous (AV) graft infections occurring at their institution during a 4.5year period. They analyzed immediate complications and long-term consequences, including the need for subsequent vascular-access procedures and duration of catheterdependent dialysis therapy.

Ninety graft infections were identified in 78 patients, yielding a rate of 8.2 infections per 100 graft-years. Patients with graft infection were much more likely to have a low serum albumin level $(<3.5 \mathrm{~g} / \mathrm{dL})$ in the month preceding the infection compared with noninfected control subjects ( $73 \%$ vs $18 \% ; P<.001)$. Infections occurred within 1 month of graft placement in $15 \%$, at 1 to 12 months in $44 \%$, and more than 1 year from surgery in $41 \%$. The pathogen was a 